\title{
Enhanced antitumor activity by the combination of dasatinib and combretastatin A-4 in vitro and in vivo
}

\author{
CHONG ZHANG $^{1}$, SHUANG-SHUANG ZHOU ${ }^{1}$, XIANG-RONG LI ${ }^{1}$, BAO-MING WANG ${ }^{1}$, NENG-MING LIN ${ }^{2}$, \\ LIN-YI FENG ${ }^{1}$, DA-YONG ZHANG ${ }^{1}$, LI-HUANG ZHANG ${ }^{1}$, JUN-BO WANG ${ }^{1}$ and JIAN-PING PAN ${ }^{1}$ \\ ${ }^{1}$ School of Medicine, Zhejiang University City College, Hangzhou, Zhejiang 310015; \\ ${ }^{2}$ Laboratory of Clinical Pharmacy, Zhejiang Cancer Hospital, Hangzhou, Zhejiang 310022, P.R. China
}

Received December 14, 2012; Accepted March 18, 2013

DOI: $10.3892 /$ or.2013.2405

\begin{abstract}
The present study showed that the combination of dasatinib and combretastatin A-4 (CA-4) exhibited synergistic cytotoxicity in multiple types of cancer, including ovarian, hepatocellular, lung and prostate carcinoma. The enhanced apoptosis induced by dasatinib plus CA-4 was accompanied by a greater extent of mitochondrial depolarization, caspase-3 activation and PARP cleavage in HO-8910 cells. Furthermore, elevated expression of Mcl-1 led to a reduced apoptosis induced by dasatinib plus CA-4, highlighting that downregulated Mcl-1 was necessary for the potentiating effect of dasatinib to CA-4-triggered apoptosis. A clear increase in $\gamma$-H2AX expression was observed in the dasatinib + CA-4 group compared with the mono-treatment groups, indicating that dasatinib plus CA-4 may induce double-strand breaks (DSBs) in HO-8910 cells. Moreover, the increased anticancer efficacy of dasatinib combined with CA-4 was further validated in a human HO-8910 ovarian cancer xenograft model in nude mice. Our study is the first to show that the combination of dasatinib with CA- 4 could be a novel and promising therapeutic approach for the treatment of cancer.
\end{abstract}

\section{Introduction}

Combretastatin A-4 (CA-4), a natural product isolated from the bark of a south african tree combretum caffrum, is a highly effective antiangiogenic agent that causes vascular shutdown, leading to tumor death (1). CA-4 phosphate (CA-4P), a water soluble pro-drug of CA-4, is rapidly dephosphorylated to the active compound CA-4 and shows reversible binding kinetics to tubulin, leading to disruption of microtubular

Correspondence to: Dr Chong Zhang or Dr Jian-Ping Pan, School of Medicine and Life Science, Zhejiang University City College, 51 Huzhou Street, Hangzhou, Zhejiang 310015, P.R. China

E-mail: zhangchong@zucc.edu.cn

E-mail:.jppan@zucc.edu.cn

Key words: combretastatin A-4, dasatinib, combination therapy, apoptosis, DNA damage structures $(2,3)$. Although CA-4P is being studied in clinical trials as a vascular disrupting agent, cardiovascular toxicity and neurotoxicity are dose limiting for CA-4P $(4,5)$. These severe side-effects currently represent the main obstacles to broad clinical application of CA-4P (2). Thus, it is important to develop new antitumor combination therapy with lower concentrations of CA-4 and more specificity for tumor endothelial cells than normal endothelial cells to avoid cardiac toxicity from endothelial damage.

Tyrosine kinase inhibitors (TKIs) are rapidly being integrated into the management of a variety of malignant diseases (6). Dasatinib, a novel orally bioactive TKI currently used to treat patients with hematologic and solid malignancies, correlated with a combined targeting of PDGFR- $\beta$ and VEGFR/c-Src signaling pathways (7-9). The Src kinases have multiple substrates that lead to diverse biological effects in cancer cells, including changes in proliferation, motility, invasion, survival and angiogenesis (10). Dasatinib suppresses tumor angiogenesis, invasion, and metastasis by inhibiting $\mathrm{Src}$ expression (11). Cardiovascular and hematologic toxicity are dose limiting for dasatinib; thus, it is necessary to develop new anticancer combination therapy with lower concentrations of dasatinib to avoid these major side-effects (12). Dasatinib has shown synergistic anticancer activity in combination with chemotherapeutic agents including paclitaxel, ixabepilone and erlotinib (12-14).

The tumor vasculature is critical to both the survival of a solid tumor mass and its continued growth (15). Angiogenesis is a complex process that occurs in a variety of physiologic and pathophysiologic states and is a remodeling of an established primitive network of blood vessels. Angiogenesis is a complex process that is essential for growth, invasion and metastasis of tumors (16). Vascular targeting agents (VTAs) comprise a novel class of anticancer agents which can be divided into two groups; those that inhibit angiogenesis (angiogenesis inhibitors) and those that target established vessels (vascular disrupting agents) (17). Various combinations involving an antiangiogenic agent and an antivascular agent have shown considerable promise in preclinical models and such combinations are currently beginning to be evaluated in patients $(18,19)$. Thus, we hypothesized that combining the antiangiogenic drug dasatinib with the antivascular agent CA- 4 might potentially enhance the anticancer therapeutic effects. 
In the present study, we showed for the first time that dasatinib and CA-4 in combination had substantial synergistic antitumor efficacy against human cancer cells in vitro and in vivo. In addition, dasatinib greatly enhanced CA-4-mediated apoptosis in HO-8910 cells, accompanied by increased extent of mitochondrial depolarization, cleavage of PARP and activation of caspase cascades. Notably, our results demonstrated that dasatinib plus CA-4 leads to increased levels of $\gamma$-H2AX indicating increased DNA damage in HO-8910 cells. These data suggested that the combination of dasatinib and CA-4 might be an effective therapeutic strategy to achieve synergistic activities in patients with solid tumors. Chemical structures of the agents are shown in Fig. 1A.

\section{Materials and methods}

Materials. CA-4 was synthesized at the Department of Chemical and Biochemical Engineering, Zhejiang University. Dasatinib was supplied by LC Laboratories (Woburn, MA, USA). The primary antibodies against Mcl-1 (S-19), Bax ( $\Delta 21)$, Bcl-2 (C-2), PARP (H250), pro-caspase-3 (H-277), XIAP (A-7), $\gamma$-H2AX (Ser139), and HRP-labeled secondary antimouse and anti-rabbit antibodies were purchased from Santa Cruz Biotechnology (Santa Cruz, CA, USA); cytochrome $c$ (136F3), cleaved-caspase-3 (D-175) were purchased from Cell Signaling Technology (Danvers, MA, USA); $\beta$-actin was purchased from BD Biosciences (Franklin Lakes, NJ, USA).

Cell culture. Human ovarian cancer cell line (HO-8910), human hepatocellular carcinoma cell line (HepG2, SMMC-7721), human non-small cell lung cancer cell line (A549) and human prostate cancer cell line (PC-3) were purchased from the Shanghai Institute of Biochemistry and Cell Biology (Shanghai, China); they were tested and authenticated for genotypes by DNA fingerprinting. HO-8910 and HepG2 were maintained in Dulbecco's modified Eagle's medium (DMEM) supplemented with $10 \%$ fetal bovine serum (FBS). A549 and PC-3 were grown in Ham's F12 medium supplemented with $10 \%$ FBS, and SMMC-7721 was cultured in RPMI-1640 medium supplemented with $10 \%$ FBS. All the cells were maintained in a humidified atmosphere of $95 \%$ air plus $5 \% \mathrm{CO}_{2}$ at $37^{\circ} \mathrm{C}$.

Cytotoxicity assay. The antiproliferative activity of combination treatment with dasatinib and CA- 4 was measured by sulforhodamine blue (SRB) cytotoxicity assay. Briefly, cells were fixed with $10 \%$ TCA solution for $1 \mathrm{~h}$, wells were rinsed 5 times with tap water and then cells were stained with $0.4 \%$ SRB solution $(100 \mu \mathrm{l} /$ well) for at least $20 \mathrm{~min}$ at room temperature. Wells were rinsed with $1 \%$ acetic acid to remove unbound dye and were then left to air dry. The SRB dye was then solubilized by placing $100 \mu \mathrm{l}$ of unbuffered Tris-based solution in each well, and the absorbance was measured at $515 \mathrm{~nm}$ using a multi-scan spectrum. The inhibition rate of cell proliferation was calculated for each well as (A515 control cells - A515 treated cells)/A515 control cells x 100\%.

Analysis of apoptosis by propidium iodide (PI) staining. Cells $\left(4 \times 10^{5} /\right.$ well) were seeded into 6-well plates and exposed to dasatinib, CA-4 or the combination for $48 \mathrm{~h}$. Cells were harvested and washed with PBS, fixed with pre-cooled $70 \%$

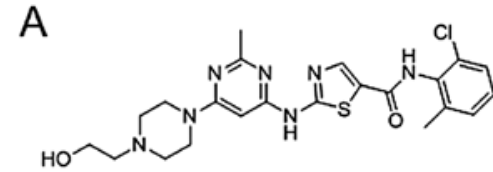

Dasatinib

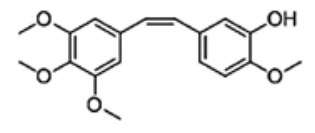

CA-4

B

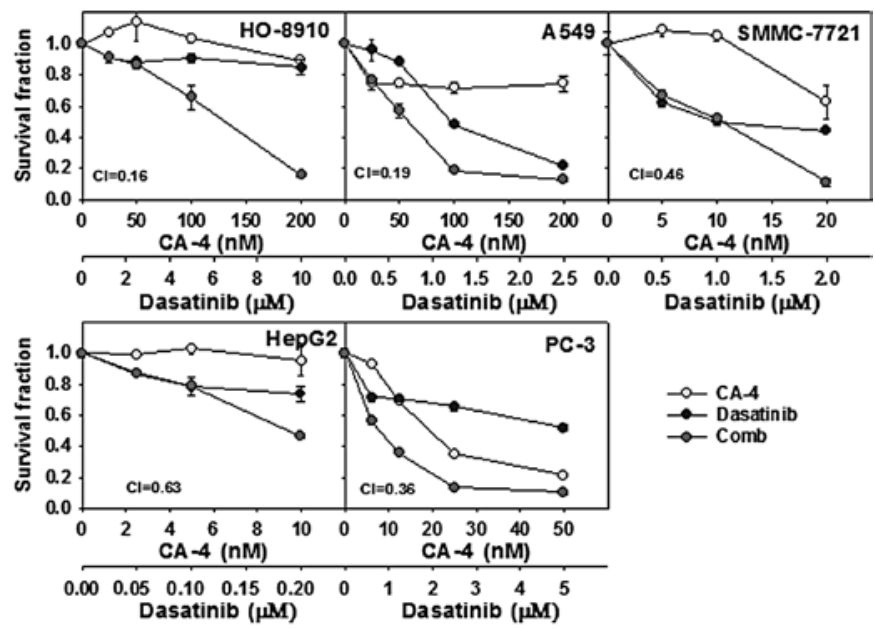

Figure 1. Cytotoxicity of the dasatinib and combretastatin A-4 (CA-4) combination. (A) Chemical structure of dasatinib and CA-4. (B) The cells were incubated with compounds for $72 \mathrm{~h}$. Dose-response curves of human cancer cell lines to dasatinib, CA-4, or the combination. Each condition had 6 replicates, and error bars represent standard deviation.

ethanol at $4^{\circ} \mathrm{C}$ overnight. Fixed cells were then washed with PBS to remove residual ethanol, pelleted, resuspended in $500 \mu \mathrm{l}$ PBS containing $50 \mu \mathrm{g}$ RNase A at $37^{\circ} \mathrm{C}$ and $5 \mu \mathrm{g}$ PI in the dark at room temperature for $30 \mathrm{~min}$. For each sample, $2 \times 10^{4}$ cells were collected and analyzed using an FACSCalibur cytometer (Becton-Dickinson, San Jose, CA, USA).

Analysis of apoptosis by DAPI staining. Briefly, cells in 96-well plates treated with dasatinib, CA-4 or the combination were washed twice with PBS, and then incubated with $0.1 \%$ Triton and $0.1 \%$ DAPI. The morphology of the cell nuclei was observed and captured using a fluorescence microscope at excitation wavelength $350 \mathrm{~nm}$.

Determination of mitochondrial membrane depolarization. Cells $\left(4 \times 10^{5} /\right.$ well $)$ were exposed to dasatinib, CA- 4 or the combination for $48 \mathrm{~h}$. They were then collected and resuspended

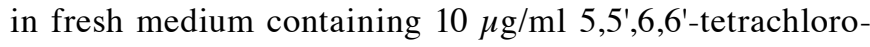
1,1'3,3'-tetraethylbenzimidazol-carbocyanine iodide (JC-1). Following incubation at $37^{\circ} \mathrm{C}$ for $30 \mathrm{~min}$, cells were analyzed by flow cytometry (20).

Cell lysates and western blot analysis. Proteins were extracted with lysis buffer $(50 \mathrm{mM}$ Tris- $\mathrm{HCl}, 150 \mathrm{mM} \mathrm{NaCl}, 1 \mathrm{mM}$ EDTA, 0.1\% SDS, $0.5 \%$ deoxycholic acid, $0.02 \%$ sodium azide, $1 \% \mathrm{NP}-40,2.0 \mu \mathrm{g} / \mathrm{ml}$ aprotinin, $1 \mathrm{mM}$ phenylmethylsulfonylfluoride). The lysates were centrifuged at $10,000 \mathrm{x} \mathrm{g}$ for $30 \mathrm{~min}$ at $4^{\circ} \mathrm{C}$. The supernatants were transferred to a new tube and the protein concentration was determined. To analyze cytochrome $c$ release from mitochondria, mitochondria was 
extracted using Mitochondria/Cytosol Fractionation kit (Beyotime Institute of Biotechnology, Haimen, China). Proteins were fractionated on 8-15\% Tris-glycine gels, and they were then transferred to PVDF membranes (Millipore, Bedford, MA, USA) and probed with primary antibodies (dilution range 1:500-1:1,000) followed by horseradish peroxidase-labeled secondary antibodies at 1:5,000 dilution. Antibody binding was then detected with the use of a chemiluminescent substrate and visualized on autoradiography film (21).

Immunofluorescence. Cells were seeded onto 35-mm dishes and exposed to dasatinib, CA-4 or the combination for $48 \mathrm{~h}$. Subsequently, the cells were fixed with $4 \%$ formaldehyde for 15 min. After washing with PBS, the cells were blocked with $10 \%$ serum in PBS for $10 \mathrm{~min}$ and incubated at $37^{\circ} \mathrm{C}$ for $2 \mathrm{~h}$ with $\gamma$-H2AX (1:200; Santa Cruz Biotechnology). The cells were then washed and incubated in the dark for $1 \mathrm{~h}$ at $37^{\circ} \mathrm{C}$ with goat anti-rabbit (FITC)-conjugated antibodies (1:200; Earthbox, San Francisco, CA, USA). After washing, the nuclei were counterstained with DAPI and the cells were then washed in PBS and examined using a laser-scanning confocal microscopy (FluoView, Olympus, Tokyo).

Plasmid transfection. pTOPO-Mcl-1 plasmid from Addgene (22) (Plasmid 21605, Addgene, Cambridge, MA, USA) or the empty vector was transfected into cells using Lipofectamine 2000 as recommended by the manufacturer.

Animals and antitumor activity in vivo. Human ovarian cancer HO-8910 xenografts were established by injecting $5 \times 10^{6}$ cells subcutaneously into nude mice. When the tumor reached a volume of $50-150 \mathrm{~mm}^{3}$, the mice were randomized to control and treated groups, and received vehicle $(0.9 \%$ sodium chloride, i.v. administration), CA-4 (6 mg/kg, 1\% DMSO, 7\% cremophor/ethanol (3:1), and 92\% sodium chloride, i.p. administration), dasatinib ( $5 \mathrm{mg} / \mathrm{kg}$, i.g. administration) 4 times per week for 19 days. Mouse weight and tumor volume were measured individually twice per week with microcalipers until animals were sacrificed. Tumor volume (V) was calculated as $\mathrm{V}=$ (length $\mathrm{x}$ width $\mathrm{x}$ high)/2. The tumor volume at day $\mathrm{n}$ was expressed as RTV according to the following formula: RTV = TVn/TV0, where TVn is the tumor volume at day $n$ and TV0 is the tumor volume at day 0 . Therapeutic effects of treatment were expressed in terms of $\mathrm{T} / \mathrm{C} \%$ using the calculation formula $\mathrm{T} / \mathrm{C}(\%)=$ mean RTV of the treated group/mean RTV of the control group x $100 \%$.

Statistical analyses. Two tailed Student's t-tests were used to determine the significance of differences between the experiment conditions. Differences were considered statistically significant at $\mathrm{P}<0.05$. Combination index (18) was well-accepted for quantifying drug synergism based on the multiple drug-effect equation of Chou-Talalay $(23,24)$. For in vitro experiments, CI values were calculated for each concentration of dasatinib, CA-4 and the combination in cell proliferation assays using Calcusyn (Biosoft, Cambridge, UK). Different CI values were obtained when solving the equation for different effect levels, and mean CI values were chosen for presentation. A $\mathrm{CI}<0.9$ indicated synergism; 0.1, very strong synergism; 0.1-0.3, strong synergism; 0.3-0.7, synergism;
0.7-0.85, moderate synergism; 0.85-0.9, slight synergism; $0.9-1.10$, additive; and $>1.10$, antagonism.

\section{Results}

Cytotoxicity of the dasatinib and CA-4 combination in human cancer cell lines. Firstly, the cytotoxicity was determined using SRB assay, after a 72-h exposure to dasatinib, CA-4 and the combination at the indicated concentration in 5 human cancer cell lines. Survival curves to dasatinib, CA-4, and dasatinib combined with CA-4 are shown in Fig. 1B. CI values were calculated using Calcusyn at the fixed-ratio concentrations of dasatinib and CA-4 to assess combination activity. Dasatinib plus CA-4 showed synergy in HO-8910, A549, SMMC-7721, HepG2 and PC-3 cell lines, with the mean CI values $<0.7$ (Fig. 1B).

Dasatinib synergizes with CA-4 to trigger apoptosis. To explore the mechanism of synergistic effects by combining dasatinib and CA-4, we first detected apoptosis by PI staining in HO-8910 and A549 cells that showed strong synergistic effects in the cytotoxicity assay. As shown in Fig. 2A, PI staining for sub-G1 content analysis was used to characterize the apoptosis in HO-8910 cells treated with $10 \mu \mathrm{M}$ dasatinib, $200 \mathrm{nM} \mathrm{CA}-4$ or the combination for $48 \mathrm{~h}$. The percentage of apoptotic cells was $4.88 \%$ in control cells, $8.16 \%$ with dasatinib, $14.30 \%$ with CA-4 and $62.92 \%$ in the combination treatment group. The difference of apoptotic cells between combination vs. monotreated groups reached statistical significant in HO-8910 and A549 cells $(\mathrm{P}<0.01)$ (Fig. 2B and C).

Dasatinib plus CA-4 induces apoptosis via the mitochondrial pathway. To further confirm the combination effect of dasatinib and CA-4 on the induction of the mitochondrial apoptosis pathway, we next detected mitochondrial membrane potential by JC-1 staining in HO-8910 and A549 cells. HO-8910 cells were treated with $10 \mu \mathrm{M}$ dasatinib, $200 \mathrm{nM}$ CA-4 or the combination for $48 \mathrm{~h}$. As demonstrated in Fig. 2A, combined treatment with dasatinib and CA-4 resulted in an increased percentage of mitochondrial membrane depolarized HO-8910 cells than either single agent $(61.98 \%$ in combination-treated cells, $15.71 \%$ in dasatinib-treated cells, $22.50 \%$ in CA-4-treated cells and $4.99 \%$ in the control group). In addition, dasatinib + CA-4 resulted in increased mitochondrial membrane potential than either drug alone in HO-8910 $(\mathrm{P}<0.01)$ and A549 cells $(\mathrm{P}<0.05)$ (Fig. 2D and E). Furthermore, DAPI staining was also performed to visualize the apoptosis by assessing chromatin condensation. As shown in Fig. 3A, $10 \mu \mathrm{M}$ dasatinib plus $200 \mathrm{nM} \mathrm{CA}-4$ triggered more apoptosis than the mono-treatment in HO-8910 cells, as indicated by the apoptotic bodies.

Dasatinib plus CA-4 combination therapy causes mitochondrial release of proapoptotic molecules for activation of proteases leading to substrate cleavage. Since caspase-3/PARP play a key role in the initiation of cellular events during the apoptotic process, we next examined the effect of dasatinib, CA-4, and the combination on the activation of caspase-3, cleavage of PARP. As shown in Fig. 3B, we found that although dasatinib and CA-4 had slight effect on caspase-3 and PARP, the two together were highly effective, inducing more signifi- 

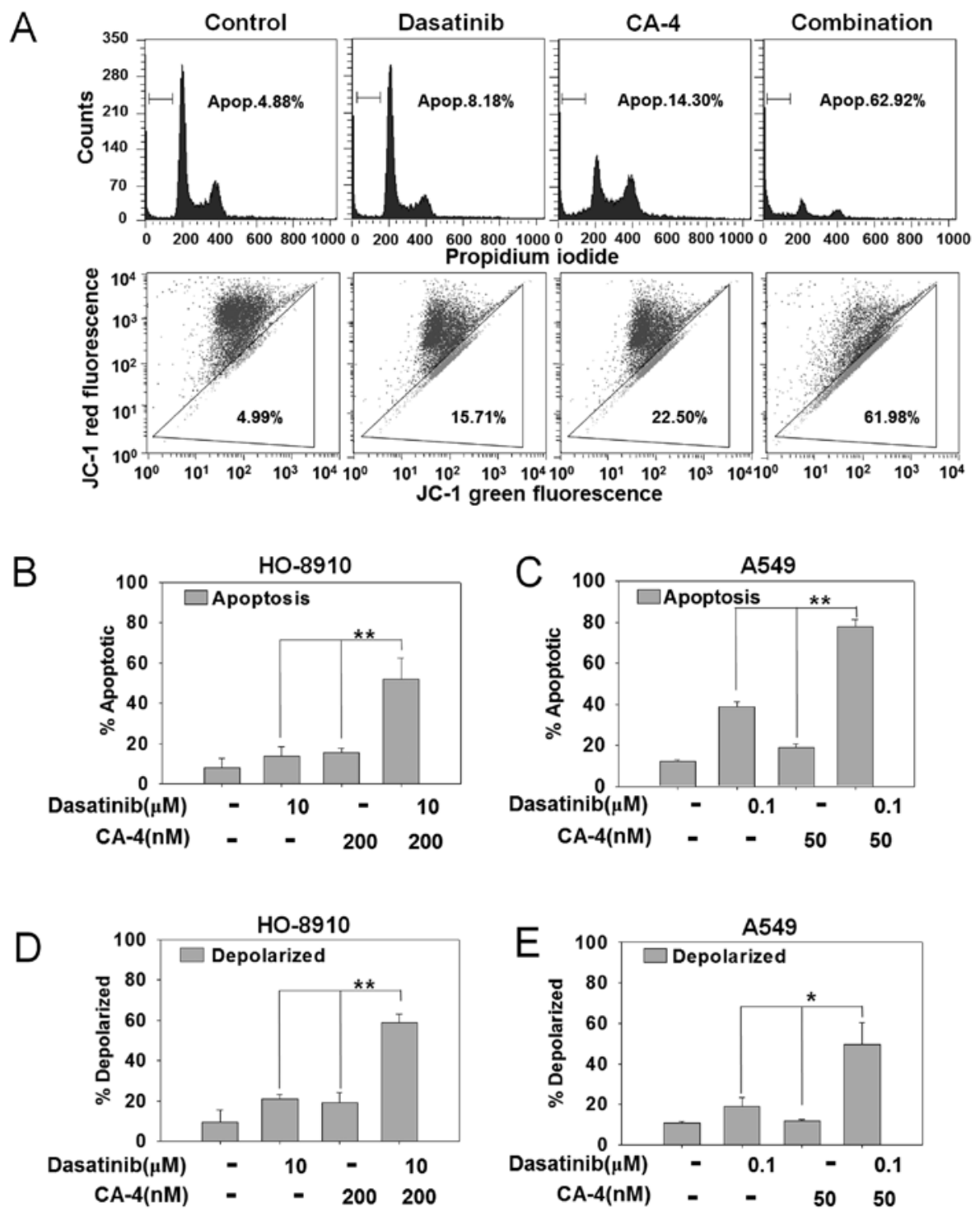

Figure 2. Dasatinib plus combretastatin A-4 (CA-4) causes enhanced apoptosis. (A) HO-8910 cells were treated with dasatinib (10 $\mu$ M), CA-4 (200 nM) or the combination for $48 \mathrm{~h}$. The cells were analyzed for apoptosis by flow cytometry and Sub-G1 contents were designed as apoptotic cells. (B) HO-8910 and (C) A549 cells in 6-well plates were exposed to compounds for $48 \mathrm{~h}$ and the cells were then analyzed by flow cytometry following PI staining. (D) HO-8910 and (E) A549 cells in 6-well plates were exposed to compounds for $48 \mathrm{~h}$ and the cells were then analyzed by flow cytometry following JC-1 staining. The experiments were repeated three times, and error bars represent standard deviation. ${ }^{*} \mathrm{P}<0.05^{* *} \mathrm{P}<0.01$.

cant cleavage of PARP and caspase-3. The X-linked inhibitor of apoptosis (XIAP) is the most potent caspase inhibitor in the IAP family and inhibits apoptotic cell death predominantly by preventing activation of initiator caspase-9 as well as effectors caspases-3 and -7 (25). Treatment of the cells with dasatinib plus CA-4 caused a significantly greater reduction of XIAP than either agent used alone. Moreover, dasatinib plus CA-4 combination treatment resulted in the decrease of Bcl-2 and increase of Bax, with an increase in the $\mathrm{Bax} / \mathrm{Bcl}-2$ ratio (Fig. 3B). To further confirm the combination effect of dasatinib and CA-4 on the induction of the mitochondrial apoptosis pathway, we also examined cytochrome $c$ release from mitochondria to cytosol. The levels of mitochondrial cytochrome $c$ were lower in cells treated with dasatinib + CA-4 than in cells treated with dasatinib or CA-4 as single agents (Fig. 3C). Collectively, these results demonstrated that the dasatinib plus CA-4 combination therapy caused mitochondrial release of cytochrome $c$ for activation of proteases leading to substrate cleavage.

Mcl-1 overexpression rescues cells from synergistic killing by the combination of dasatinib and CA-4. We found that Mcl-1 expression was markedly downregulated in dasatinib plus CA-4 combination-treated cells compared with single agent alone, indicating that Mcl-1 might be involved in the synergistic effect of the combination treatment (Fig. 3B). To further analyze whether Mcl-1 downregulation was required for dasatinib plus CA-4 combination treatment-induced apoptosis, we performed Mcl-1 overexpression experiments. We successfully increased the protein expression of Mcl-1 in HO-8910 cells by transfecting with pTOPO-Mcl-1 plasmid (Fig. 3D). The decrease of pro-caspase-3 caused by dasatinib plus CA-4 was also increased due to the elevated levels of Mcl-1 in the cells (Fig. 3E). Our observations indicate that the downregulation 
A

B
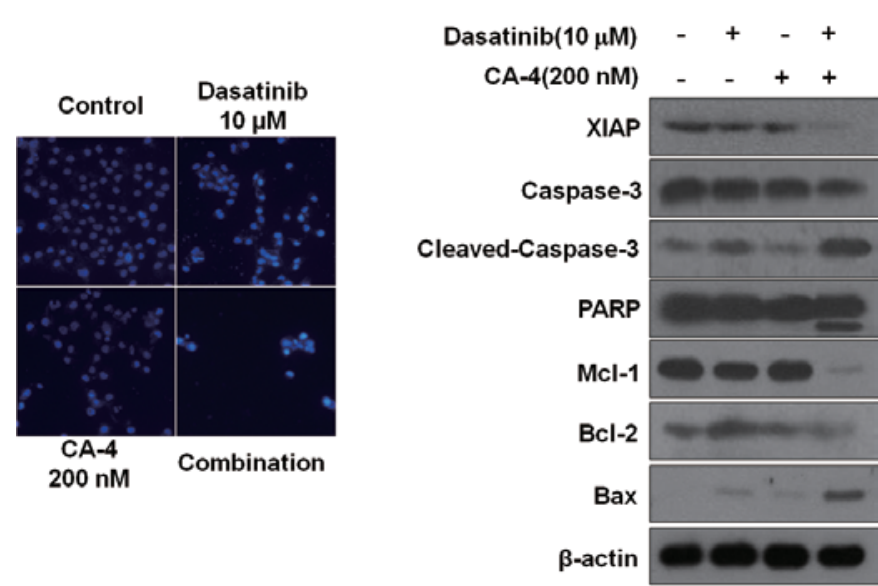

C

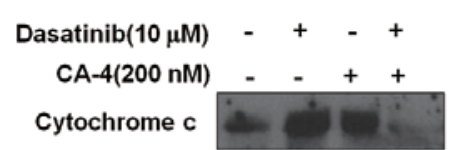

D

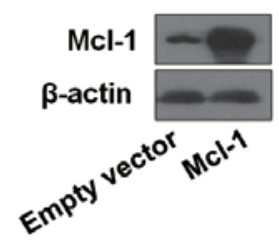

E

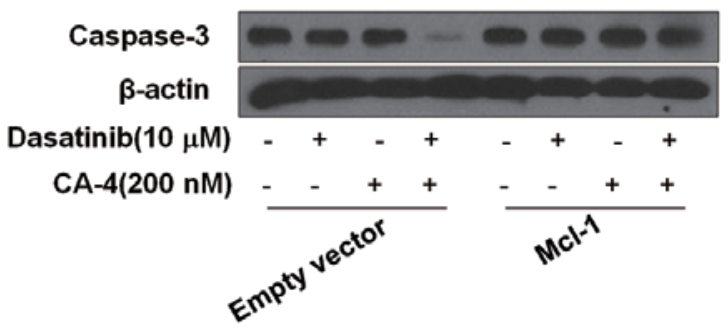

Figure 3. Dasatinib plus combretastatin A-4 (CA-4) causes activation of various apoptosis-related proteins. (A) Dasatinib plus CA-4 induced apoptotic bodies in HO-8910 cells. Nuclear DNA was visualized by DAPI staining. (B) HO-8910 cells were exposed to dasatinib $(10 \mu \mathrm{M})$, CA-4 $(200 \mathrm{nM})$ or the combination for $48 \mathrm{~h}$, after which protein extracts were immunoblotted with specified antibodies for XIAP, caspase-3, cleaved-caspase-3, PARP, Mcl-1, Bcl-2, Bax and $\beta$-actin. (C) Cytochrome $c$ release from mitochondria into cytosol was assessed at $48 \mathrm{~h}$ of incubation with the agents. (D) HO-8910 cells were transfected with Mcl-1 plasmid and empty vector according to the manufacturer's recommendations. Forty-eight hours after transfection, cell lysates were prepared for western blot analysis. (E) The expression of caspase-3 in HO-8910 cells that had been transfected with Mcl-1 plasmid or empty vector and then treated with $10 \mu \mathrm{M}$ dasatinib, either alone or in combination with $200 \mathrm{nM} \mathrm{CA}-4$ for $48 \mathrm{~h}$, were examined.

of Mcl-1 may contribute to the synergistic effect of dasatinib and $\mathrm{CA}-4$.

Dasatinib in combination with CA-4 induces DNA damage. The induction of phosphorylated $\mathrm{H} 2 \mathrm{AX}(\gamma-\mathrm{H} 2 \mathrm{AX})$ is a marker of DNA damage. Next, we determined whether dasatinib in combination with CA- 4 could induce DNA damage in HO-8910 cells. As shown in Fig. 4A, the induction of $\gamma$-H2AX in HO-8910 cells was observed by western blot analysis, only in the combination setting after $6 \mathrm{~h}$, indicating DNA damage might be involved in the synergistic effect of dasatinib and
A

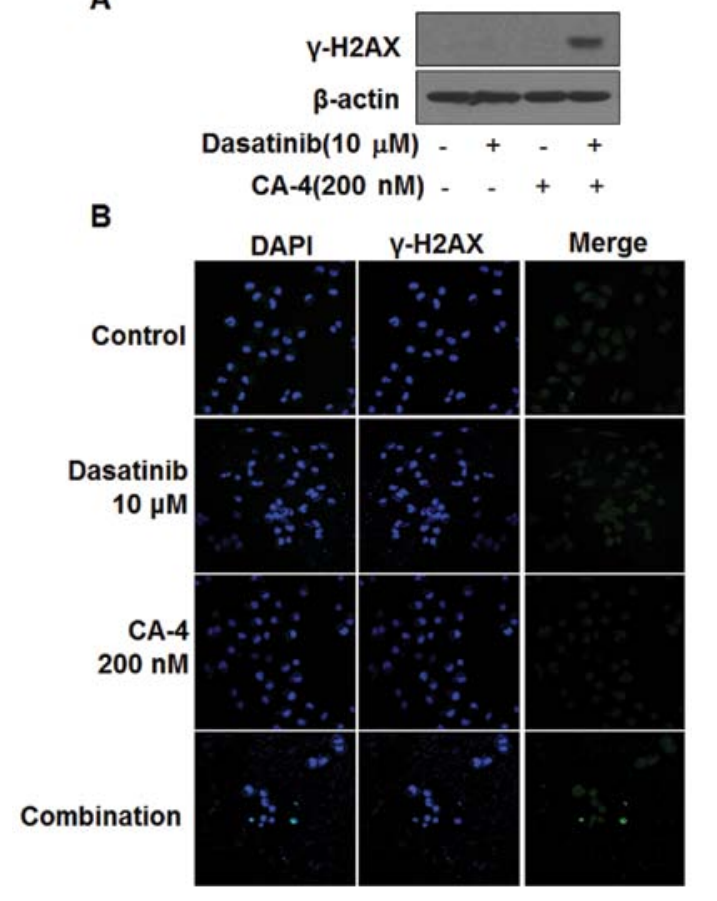

Figure 4. (A) Dasatinib in combination with combretastatin A-4 (CA-4) induces DNA damage. HO-8910 cells were exposed to dasatinib $(10 \mu \mathrm{M})$, CA-4 $(200 \mathrm{nM})$ or the combination for $6 \mathrm{~h}$, after which protein extracts were immunoblotted using $\gamma-\mathrm{H} 2 \mathrm{AX}$. (B) $\mathrm{HO}-8910$ cells were treated with dasatinib $(10 \mu \mathrm{M}), \mathrm{CA}-4(200 \mathrm{nM})$ or the combination for $6 \mathrm{~h}$ and imaged as in a stained with $\gamma$-H2AX immunofluorescence, DAPI (blue). $\gamma$-H2AX expression was indicated by a stippled appearance in the nucleus. Scale bar, $40 \mu \mathrm{m}$.

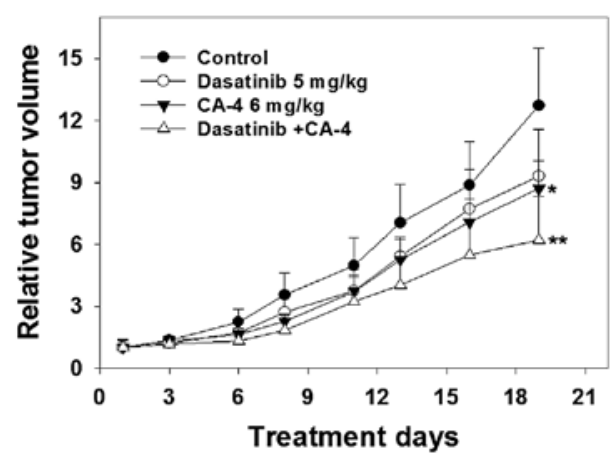

Figure 5. The synergistic effect of dasatinib and combretastatin A-4 (CA-4) on the HO-8910 human xenograft model. The mice transplanted with HO-8910 human xenografts were randomly divided into four groups and administered injection of dasatinib ( $5 \mathrm{mg} / \mathrm{kg}$, i.g.), CA-4 (6 mg/kg, i.p.), the combination or vehicle daily for a period of 19 days. Relative tumor volume is expressed as mean $\pm \mathrm{SD}$ ( $\mathrm{n}=10$ per group).

CA-4. Furthermore, immunofluorescence results showed the number of $\gamma$-H2AX-positive cells was significantly increased in HO-8910 cells treated with dasatinib and CA-4, but not in the mono-treatment groups (Fig. 4B).

The antitumor activity of dasatinib and CA-4 combination therapy against human $\mathrm{HO}-8910$ xenografts. To further characterize the anticancer efficacy of combination treatment, the in vivo activity of dasatinib and CA-4 was tested on an ovarian cancer HO-8910 xenograft model in nude mice. As shown in Fig. 5 and Table I, the i.g. administration of dasatinib at a 
Table I. In vivo efficacy of dasatinib in combination with CA-4 against HO-8910 xenografts.

\begin{tabular}{|c|c|c|c|c|c|c|}
\hline \multirow[b]{2}{*}{ Group } & \multicolumn{2}{|c|}{ No. of animals } & \multicolumn{2}{|c|}{ Body weight $(\mathrm{g})$} & \multirow[b]{2}{*}{ Mean RTV } & \multirow[b]{2}{*}{$\mathrm{T} / \mathrm{C}(100 \%)$} \\
\hline & Start & End & Start & End & & \\
\hline Control & 10 & 10 & $20.6 \pm 0.6$ & $23.4 \pm 0.7$ & 12.7 & - \\
\hline Dasatinib $(5 \mathrm{mg} / \mathrm{kg})$ & 10 & 10 & $20.7 \pm 1.5$ & $22.7 \pm 1.1$ & 9.3 & 73.2 \\
\hline CA-4 (6 mg/kg) & 10 & 10 & $20.2 \pm 1.4$ & $21.7 \pm 1.1$ & $8.7^{\mathrm{a}}$ & 68.5 \\
\hline Combination & 10 & 10 & $20.5 \pm 0.6$ & $20.5 \pm 0.6$ & $6.2^{\mathrm{b}, \mathrm{c}, \mathrm{d}}$ & 48.8 \\
\hline
\end{tabular}

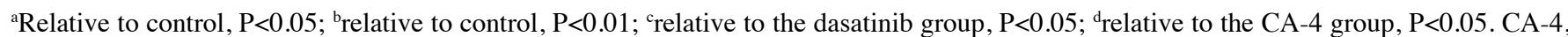
combretastatin A-4.

dose of $5 \mathrm{mg} / \mathrm{kg}$ four times every week for 19 days produced slight antitumor effect in mean RTV compared with that of the control group (mean RTV, CA-4 vs. control: 9.3 vs. 12.7; $\mathrm{P}>0.05$ ). However, with the dosage of $6 \mathrm{mg} / \mathrm{kg}$ four times every week for 19 days, CA-4 exerted a moderate tumor growth inhibitory effect (mean RTV, CA-4 vs. control: 8.7 vs. 12.7; $\mathrm{P}<0.05)$. As expected, dasatinib plus CA-4 caused marked tumor growth inhibition (T/C value, 48.8\%), significantly greater than CA-4- (T/C value, $68.5 \%$ ) or dasatinib treatment alone (T/C value, $73.2 \%$ ). The RTV of the combination group was markedly decreased compared to that of the control group $(\mathrm{P}<0.01)$; notably, compared with the dasatinib or the CA-4 group, the combination group exerted significantly more potent antitumor activities against the HO-8910 xenograft in nude mice.

\section{Discussion}

Dasatinib, an orally active, small molecule targeting agent that inhibits multiple members of the SRC family and other tyrosine kinases, such as PDGFR, is initially approved for use in $\mathrm{Ph}+$ acute lymphoblastic leukemia and is currently undergoing clinical trials in a variety of solid tumor types (26). Dasatinib reduces angiogenesis and metastasis through inhibiting Src family kinases (27). Inhibitors of angiogenesis interfere with new vessel formation and therefore have a preventative action, with little effect on the existing tumor blood vessels, and are likely to be of particular benefit in early-stage cancer therapy (28). Vascular-disrupting agents target the established tumor blood vessels, resulting in tumor ischemia and necrosis (29). CA-4 is the lead compound as a vasculardisrupting agent and is currently in Phase II/III clinical trials against a range of malignancies, in combination with conventional chemotherapeutic agents and radiotherapy $(30,31)$. Furthermore, CA-4 displays minimal toxicity profile at low dose, indicating its potential as a candidate for drug combinations in the solid tumor therapy $(32,33)$. Since both the initiation of new vessel formation and the integrity of the existing blood vessel network are critical to tumor growth and survival, various combinations involving an antiangiogenic agent and an antivascular agent could cause significantly more effective antitumor responses in various preclinical models (34). Thus, combining dasatinib with CA-4 may be a logical way to potentially enhance response rates and prolong survival times for patients by targeting tumor blood vessels.
The results of the present report, for the first time, indicated that the synergistic anticancer effects in vitro and in vivo achieved by dasatinib plus CA-4 were observed in human cancer cells and a xenograft nude mice model. CI values and the significant decline of the survival curves in the combination group strongly demonstrated that dasatinib potentiated the CA-4-imposed cytotoxicity in 5 human solid tumor cell lines, including ovarian cancer (HO-8910), hepatocellular carcinoma (HepG2, SMMC-7721), non-small cell lung cancer (A549) and prostate cancer (PC-3). Furthermore, the strong synergistic effect was also validated on the HO-8910 xenograft nude mice model. As single agents, dasatinib and CA-4 merely displayed insignificant activities against the HO-8910 xenograft model, respectively; by contrast, the coadministration of dasatinib and CA-4 clearly arrested tumor growth by $51.2 \%$. Moreover, the combination of dasatinib and CA-4 markedly improved the antitumor capacities in vivo without increasing toxicities, as indicated by the nearly constant body weights in the combination-treated group on day 19. As we reported, the combination of dasatinib and CA-4 might be an effective therapeutic strategy to achieve synergistic antitumor effects in patients with solid tumors.

Our data showed that synergism in vitro achieved by the combination of dasatinib and CA-4 was accompanied by enhanced apoptosis. Activation of caspase-3 leads to the cleavage of PARP, the presence of cleaved PARP is one of the most used diagnostic tools for the detection of apoptosis in several cell types (35). From western blot analysis, marked increases in cleaved-PARP and caspase- 3 were observed in HO-8910 cells following dasatinib and CA-4 combination treatment. IAPs also play an important role during the apoptotic process; XIAP is the best known member of this family, and correlates with resistance to chemotherapy (36). Our data showed that the synergistic effect on apoptosis obtained with the dasatinib/CA-4 co-treatment was accompanied by a large decrease in antiapoptotic XIAP protein. Mitochondria play a key role in apoptotic cell death by releasing the effector proteins, including cytochrome $c$ and Smac/DIABLO, from the mitochondrial intermembrane space (IMS) (37). Our results showed that loss of mitochondrial membrane potential was significantly greater with dasatinib plus CA-4 than with either drug alone, suggesting that combination therapy might activate the mitochondrial-mediated apoptosis pathway in HO-8910 cells. In addition, we observed that the combination of dasatinib and CA-4 enhanced cytochrome $c$ release from 
mitochondria into the cytosol in HO-8910 cells. IMS protein release can result from the mitochondrial outer membrane permeabilization (MOMP), which is thought to be regulated by proteins of the Bcl-2 family (38). The Bcl-2 protein family includes proapoptotic members, such as Bax, Bad and Bcl-Xs, and antiapoptotic members, such as Bcl-2, Bcl-XL and Mcl-1. Antiapoptotic members act as repressors of apoptosis by blocking the release of cytochrome $c$, whereas proapoptotic members act as promoters (39). The final action is dependent on the balance between Bcl-2 and Bax, and the increase of Bax/ $\mathrm{Bcl}-2$ ratio can induce the release of cytochrome $c$ (40). Our results demonstrated a marked increase of the $\mathrm{Bax} / \mathrm{Bcl}-2$ ratio in HO-8910 cells treated with dasatinib plus CA-4, indicating that the regulation of $\mathrm{Bcl}-2$ protein family expression plays an important role in combination-induced apoptosis. Mcl-1, primarily localized to the outer mitochondrial membrane, has been suggested to function as an antiapoptotic factor by suppressing cytochrome $c$ release from mitochondria via heterodimerization with and neutralization of effector proapoptotic Bcl-2 family members. Mcl-1 expression was not altered in either the dasatinib or the CA-4 treatment group; however, dasatinib plus CA-4 treatment significantly downregulated the expression of Mcl-1 in HO-8910 cells, indicating that Mcl-1 might be involved in the synergistic effect of combination treatment. Moreover, elevated expression of Mcl-1 led to a reduced apoptosis induced by dasatinib plus CA-4, highlighting that downregulated Mcl-1 was necessary for the potentiating effect of dasatinib to CA-4-triggered apoptosis.

Among the different forms of complex DNA damage, double-strand breaks (DSBs) are considered to be among the most lethal forms of DNA damage, severely compromising genomic stability (41). H2AX moved to the center of cellular responses to DNA damage after the discovery that it becomes locally phosphorylated on Ser139, to generate $\gamma$-H2AX, in the vicinity of DSBs (42). Quantitation of $\gamma-\mathrm{H} 2 \mathrm{AX}$ is one of the earliest events in the DNA damage signaling and repair $(43,44)$. To maintain genomic stability following DNA damage, multicellular organisms activate checkpoints that induce cell cycle arrest or apoptosis (45). A number of anticancer drugs exert their effect by causing DSBs and subsequent apoptosis induction, such as DNA replication inhibitors, crosslinking agents and topoisomerase inhibitors (46). In our study, a clear increase in $\gamma-\mathrm{H} 2 \mathrm{AX}$ expression was observed in the dasatinib + CA-4 group compared with the mono-treatment groups. Furthermore, in HO-8910 cells, the number of $\gamma$-H2AXpositive cells increased in the combination treatment group. These results indicated that dasatinib plus CA-4 might induce DSBs in HO-8910 cells, thereby activating apoptosis pathways, and finally resulting in the synergism of these two drugs.

In conclusion, we presented evidence showing enhanced therapeutic activity of dasatinib when combined with CA-4 in vitro and in vivo. The enhanced apoptosis induced by dasatinib plus CA-4 was accompanied by the larger extent of DNA damage, mitochondrial depolarization, caspase-3 activation and PARP cleavage in HO-8910 cells. Additional preclinical studies, such as antivascular efficacy, are required to systematically evaluate the applicability of this combination as a therapeutic strategy for cancer patients. As we reported, the combination of dasatinib and CA-4 might be an effective therapeutic strategy to achieve synergistic antitumor effects.

\section{Acknowledgements}

The authors thank Mr. Yong-zhou Hu of Zhejiang University for providing CA-4 for this study. The authors acknowledge the financial support from the Teachers Research Fund of Zhejiang University City College (J-12019, J-12021), the Zhejiang Provincial Foundation of National Science (Y2100682, LQ12H31001), the Science Research Foundation of Zhejiang Health Bureau (2012KYA068, 2012KYB066), the Zhejiang Provincial Program for the Cultivation of High-level Innovative Health talents (2010-190-4), the Student Research Fund of Zhejiang University City College (XZ2012562091, X2012562098) and the Scientific Research Fund of Zhejiang Provincial Education Department (Y201120633).

\section{References}

1. Jin X, Yang YD, Chen $\mathrm{K}$, et al: HDMCP uncouples yeast mitochondrial respiration and alleviates steatosis in L02 and hepG2 cells by decreasing ATP and $\mathrm{H}_{2} \mathrm{O}_{2}$ levels: a novel mechanism for NAFLD. J Hepatol 50: 1019-1028, 2009.

2. Simoni D, Romagnoli R, Baruchello R, et al: Novel A-ring and B-ring modified combretastatin A-4 (CA-4) analogues endowed with interesting cytotoxic activity. J Med Chem 51: 6211-6215, 2008.

3. Tian $\mathrm{CH}$, Zhao JF, Xu YP, et al: Involvement of Bombyx mori nucleopolyhedrovirus ORF41 (Bm41) in BV production and ODV envelopment. Virology 387: 184-192, 2009.

4. Nagaiah G and Remick SC: Combretastatin A4 phosphate: a novel vascular disrupting agent. Future Oncol 6: 1219-1228, 2010.

5. Cooney MM, Radivoyevitch T, Dowlati A, et al: Cardiovascular safety profile of combretastatin a4 phosphate in a single-dose phase I study in patients with advanced cancer. Clin Cancer Res 10: 96-100, 2004.

6. Su C, He P, Yan RJ, Zhao CB and Zhang C: Study of the orientation-controlled damping temperature based on selective distribution of oligo-phenol in acrylate rubber/chlorinated butyl rubber blends. Polymer Composites 33: 860-865, 2012.

7. Ablikim M, Achasov MN, Alberto D, et al: Search for $\eta_{c}{ }^{\prime}$ decays into vector meson pairs. Physical Review D 84: 091102, 2011

8. Li J, Li J, Li S, et al: Ameliorative effect of grape seed proanthocyanidin extract on thioacetamide-induced mouse hepatic fibrosis. Toxicol Lett 213: 353-360, 2012.

9. Zhang CL, Wang $\mathrm{H}$ and Yan J: Leptospirosis prevalence in Chinese populations in the last two decades. Microbes Infect 14: 317-323, 2012.

10. Bao YY, Xue J, Wu WJ, Wang Y, Lv ZY and Zhang CX: An immune-induced Reeler protein is involved in the Bombyx mori melanization cascade. Insect Biochem Mol Biol 41: 696-706, 2011.

11. Zhang XF, Zhang CH, Yang B, Lv N, Pan QF and Ye GX: Aggregation mechanism of $\mathrm{Ag}$ atoms deposited on liquid surfaces. J Phys Soc Jpn 80: 104603-104604, 2011.

12. Li GL, Roy B, Li XH, et al: Quantification of silkworm coactivator of MBF1 mRNA by SYBR Green I real-time RT-PCR reveals tissue- and stage-specific transcription levels. Mol Biol Rep 36: 1217-1223, 2009.

13. Zhang CQ, Liu YH, Ma XY, Feng Z and Ma ZH: Characterization of sensitivity of Rhizoctonia solani, causing rice sheath blight, to mepronil and boscalid. Crop Protection 28: 381-386, 2009.

14. Ma TY, Yan M, Zhang CS, Pei YM and Jiang CB: Stress influences on magnetization and magnetostriction in magnetically annealed Tb0.36Dy0.64(Fe0.85Co0.15)(2) polycrystals. J Appl Phys 105: 093915, 2009.

15. Dark GG, Hill SA, Prise VE, Tozer GM, Pettit GR and Chaplin DJ: Combretastatin A-4, an agent that displays potent and selective toxicity toward tumor vasculature. Cancer Res 57: 1829-1834, 1997.

16. Gay LJ and Felding-Habermann B: Contribution of platelets to tumour metastasis. Nat Rev Cancer 11: 123-134, 2011.

17. Assifi MM and Hines OJ: Anti-angiogenic agents in pancreatic cancer: a review. Anticancer Agents Med Chem 11: 464-469, 2011. 
18. Pasquier E, Ciccolini J, Carre M, et al: Propranolol potentiates the anti-angiogenic effects and anti-tumor efficacy of chemotherapy agents: implication in breast cancer treatment. Oncotarget 2: 797-809, 2011

19. Fang ZN, Yang B, Chen MG, Zhang CH, Xie JP and Ye GX: Growth and morphology of ultra-thin Al films on liquid substrates studied by atomic force microscopy. Thin Solid Films 517: 3408-3411, 2009.

20. Cao J, Xu D, Wang D, et al: ROS-driven Akt dephosphorylation at Ser-473 is involved in 4-HPR-mediated apoptosis in NB4 cells. Free Radic Biol Med 47: 536-547, 2009.

21. Liu XW, Su Y, Zhu H, et al: HIF-1 $\alpha$-dependent autophagy protects HeLa cells from fenretinide (4-HPR)-induced apoptosis in hypoxia. Pharmacol Res 62: 416-425, 2010.

22. Wang C, Wang JF, Chen C, et al: Learning to extract web news title in template independent way. In: Rough Sets and Knowledge Technology Proceedings Gold Coast. Springer, Berlin, pp192$199,2009$.

23. Chou TC and Talalay P: Generalized equations for the analysis of inhibitions of Michaelis-Menten and higher-order kinetic systems with two or more mutually exclusive and nonexclusive inhibitors. Eur J Biochem 115: 207-216, 1981.

24. Chou TC and Talalay P: Quantitative analysis of dose-effect relationships: the combined effects of multiple drugs or enzyme inhibitors. Adv Enzyme Regul 22: 27-55, 1984.

25. Salvesen GS and Duckett CS: IAP proteins: blocking the road to death's door. Nat Rev Mol Cell Biol 3: 401-410, 2002.

26. Zhang CL, Feng LF, Hoppe $\mathrm{S}$ and $\mathrm{Hu} \mathrm{GH}$ : Residence time distribution: an old concept in chemical engineering and a new application in polymer processing. AIChE J 55: 279-283, 2009.

27. Zhang CL, Chen WQ and Yang JS: Electrically forced vibration of a rectangular piezoelectric plate of monoclinic crystals. Int J Appl Electrom Mechanics 31: 207-218, 2009.

28. Osusky KL, Hallahan DE, Fu A, Ye F, Shyr Y and Geng L: The receptor tyrosine kinase inhibitor SU11248 impedes endothelial cell migration, tubule formation, and blood vessel formation in vivo, but has little effect on existing tumor vessels. Angiogenesis 7: 225-233, 2004.

29. Wu D, Zhu DS, He Y, Zhang CQ and Feng L: Nondestructive detection of grey mold of eggplant based on ground multispectral imaging sensor. Guang Pu Xue Yu Guang Pu Fen Xi 28: 1496-1500, 2008 (In Chinese).

30. Tozer GM, Kanthou C, Lewis G, Prise VE, Vojnovic B and Hill SA: Tumour vascular disrupting agents: combating treatment resistance. Br J Radiol 81: S12-S20, 2008.

31. Wu R, Ding W, Liu T, et al: XN05, a novel synthesized microtubule inhibitor, exhibits potent activity against human carcinoma cells in vitro. Cancer Lett 285: 13-22, 2009.

32. Busk M, Bohn AB, Skals M, Wang T and Horsman MR: Combretastatin-induced hypertension and the consequences for its combination with other therapies. Vascul Pharmacol 54: 13-17, 2011.
33. Zhu H, Zhang J, Xue N, Hu Y, Yang B and He Q: Novel combretastatin A-4 derivative XN0502 induces cell cycle arrest and apoptosis in A549 cells. Invest New Drugs 28: 493-501, 2010.

34. Siemann DW, Chaplin DJ and Horsman MR: Vascular-targeting therapies for treatment of malignant disease. Cancer 100: 2491-2499, 2004.

35. Narula J, Pandey P, Arbustini E, et al: Apoptosis in heart failure: release of cytochrome $\mathrm{c}$ from mitochondria and activation of caspase-3 in human cardiomyopathy. Proc Natl Acad Sci USA 96: 8144-8149, 1999.

36. Kashkar H: X-linked inhibitor of apoptosis: a chemoresistance factor or a hollow promise. Clin Cancer Res 16: 4496-4502, 2010.

37. Zhao GL, Li H, Zhang CS and Han GR: Preparation of $\mathrm{TiO}_{2}$ films under low temperature condition on the glass substrate. Rare Metal Mat Eng 37: 148-150, 2008.

38. Xu HJ, Yang ZN, Zhao JF, et al: Bombyx mori nucleopolyhedrovirus ORF56 encodes an occlusion-derived virus protein and is not essential for budded virus production. J Gen Virol 89: 1212-1219, 2008

39. Ablikim M, Bai JZ, Ban Y, et al: Determination of the psi (3770), psi (4040), psi (4160) and psi (4415) resonance parameters. Physics Lett B 660: 315-319, 2008.

40. Saxena P and Tesar PJ: Vascular obstruction related to mediastinal fibrosis: an interesting clinical entity. J Thorac Cardiovasc Surg 134: 1379-1380, 2007.

41. Kinner A, Wu W, Staudt C and Iliakis G: $\gamma-\mathrm{H} 2 \mathrm{AX}$ in recognition and signaling of DNA double-strand breaks in the context of chromatin. Nucleic Acids Res 36: 5678-5694, 2008.

42. Mah LJ, El-Osta A and Karagiannis TC: $\gamma \mathrm{H} 2 \mathrm{AX}$ : a sensitive molecular marker of DNA damage and repair. Leukemia 24: 679-686, 2010.

43. Smart DJ, Ahmedi KP, Harvey JS and Lynch AM: Genotoxicity screening via the $\gamma \mathrm{H} 2 \mathrm{AX}$ by flow assay. Mutat Res 715: 25-31, 2011.

44. Zhang DY, Pan Y, Zhang $C$, et al: Wnt $/ \beta$-catenin signaling induces the aging of mesenchymal stem cells through promoting the ROS production. Mol Cell Biochem 374: 13-20, 2012.

45. Gartner A, Milstein S, Ahmed S, Hodgkin J and Hengartner MO: A conserved checkpoint pathway mediates DNA damage induced apoptosis and cell cycle arrest in C.elegans. Mol Cell 5: 435-443, 2000.

46. Kawanishi S and Hiraku Y: Amplification of anticancer drug-induced DNA damage and apoptosis by DNA-binding compounds. Curr Med Chem Anticancer Agents 4: 415-419, 2004. 